\title{
Mentoring con Energía: un programa de mentorías on-line desde el sector energético a estudiantes de la ETSIME - UPM Mentoring with Energy: an on-line mentoring program from the energy sector to students in School of Mines and Energy
}

\author{
Cristina Montalvo ${ }^{1}$, José Luis Parra ${ }^{2}$, Ana Isabel Gávez ${ }^{3}$ \\ cristina.montalvo@upm.es, joseluis.parra@upm.es,galvez.ana@gmail.com \\ ${ }^{1,2}$ Escuela de Minas y Energía \\ UPM \\ Madrid, España \\ ${ }^{3}$ Asociación Española de Mujeres de la Energía \\ AEMENER \\ Madrid, España
}

\begin{abstract}
Resumen- Este trabajo es una colaboración entre la ETSIME (UPM) y la Asociación Española de Mujeres de la Energía (AEMENER) mediante un programa de mentoring orientado a alumnos de grado y máster de últimos cursos que van a comenzar a buscar sus primeras experiencias laborales. El programa está integrado en los planes de estudio de grado como actividad acreditable de 1 ECTS. Las mentoras son profesionales del sector energético, socias de AEMENER y tienen experiencia en procesos de mentorazgo. El programa se ha extendido a lo largo de 6 meses, desde noviembre de 2020 hasta abril de 2021 y se ha compuesto de una serie de sesiones individuales mentor-mentee y de charlas grupales enfocadas a una determinada temática. El grado de satisfacción de mentoras y mentees ha sido muy alto y se seguirá realizando en cursos posteriores.
\end{abstract}

\section{Palabras clave: Mentoría, competencias transversales, soft-skills}

Abstract- This work is a collaboration between ETSIME (UPM) and the Spanish Association of Women for Energy (AEMENER) through a mentoring program meant for Bachelor and Master students who are looking for their first jobs. The program is included in the study plans as a 1 ECTS accreditable activity. The mentors are professionals from the energy sector, members of AEMENER and they have previous experience in mentoring processes. The program had a 6-month duration from November 2020 until April 2021 and it is composed of a series of individual sessions mentor-mentee and group sessions focused on a certain subject. The degree of satisfaction is very high, and the program will continue in following years.

\section{Keywords: Mentoring, soft skills, transversal competences}

\section{INTRODUCCIÓN}

Las actividades y programas de mentoring están aumentando en la educación superior, especialmente en la última década. El número de artículos de investigación vinculados a los resultados de programas de mentoring está creciendo notablemente desde 2009 tal y como se señala en (TinocoGiraldo, Torrecilla Sánchez, \& García-Peñalvo, 2020).

Según Risquex y Sanchez-García, mentoring es una herramienta que contribuye al desarrollo de estrategias de crecimiento personal y profesional en los participantes (Risquez \& Sanchez-Garcia, 2012) . Por tanto, el rol del mentor es entrenar al mentee a través de múltiples herramientas de manera que se conviertan en mejores observadores de ellos mismos y de sus relaciones (DiRenzo, Linnehan, Shao, \& Rosenberg, 2010).

Dentro de los programas de mentoring, existen varios tipos, desde estudiante a estudiante, recién graduado a estudiante, profesor-estudiante y en menor medida, programas en los que el mentor es un profesional de mucha experiencia en la industria y que orienta a un estudiante que quiere integrarse en su sector.

Siguiendo esta línea, en la Escuela Técnica Superior de Ingenieros de Minas y Energía (ETSIME) de la Universidad Politécnica de Madrid (UPM), se ha lanzado este último curso 2020/21 un programa de mentoring de 1 ECTS incluido como actividad acreditable en sus titulaciones de grado. En este caso, todas las mentoras son profesionales del sector energético y los mentees estudiantes de últimos cursos de grados de ingeniería. Es la primera vez que se lanza un programa de este estilo en la UPM y que está integrado dentro de los planes de estudio como actividad acreditable y no como parte de programas de atracción de talento que lanzan algunas empresas. Con este tipo de programas se orienta a los estudiantes a salir al mercado laboral con más confianza y se les ayuda a establecer claramente un plan de carrera de acuerdo a sus objetivos y necesidades. La asignatura de prácticas de empresa aborda parcialmente estos aspectos pero con una perspectiva global y no se centra en cada alumno de forma individual. El programa de mentoring es innovador en tanto en cuanto supone una atención individualizada a cada alumno por parte de su mentor.

Debido a la situación de la pandemia, el programa ha sido totalmente on-line $\mathrm{y}$ ha consistido en sesiones tanto individuales, mentor-mentee, como grupales. Los resultados del trabajo se basan fundamentalmente en las opiniones tanto de las mentoras como de los mentees al terminar el programa. Se ha podido comprobar a través de las encuestas que los mentees han ganado conocimiento respecto a los posibles sectores en los que pueden colocarse y las mentoras destacan el enriquecimiento personal que les ha supuesto llevar a cabo el mentoring.

\section{CONTEXTO}


La Asociación Española de Mujeres de la Energía es una organización sin ánimo de lucro que se creó en 2018 con el objetivo de conseguir que la presencia de la mujer en todas las áreas de la empresa sea equilibrada y que los equipos sean diversos, mediante el fomento de las vocaciones femeninas en las áreas de ciencias, tecnología, ingeniería y matemáticas (las llamadas STEM, su acrónimo en inglés). Uno de los objetivos de la asociación es ayudar a la empleabilidad fortaleciendo la presencia de las mujeres en el sector energético a todos los niveles, especialmente en aquellos con mayores dificultades, así como impulsar a la mujer en la promoción y desarrollo de su carrera profesional en el sector energético.

En 2019, AEMENER firmó un convenio de colaboración con la UPM para comenzar a desarrollar actividades conjuntas relacionadas con el fomento de las carreras STEM y la atracción de talento femenino a las escuelas de ingeniería.

En este contexto, se ha desarrollado la actividad de Mentoring con Energía como una metodología docente no formal. Se trata de un programa de mentorazgo de profesionales de AEMENER hacia estudiantes de la ETSIME de últimos cursos de grado o de máster con varios objetivos:

- Apoyar y guiar a estudiantes de últimos cursos a orientar su incorporación al entorno profesional

- Orientar académicamente a aquellos estudiantes que quieran continuar con su formación.

- Conocer qué competencias y habilidades demanda el mercado de trabajo

- Identificar las motivaciones personales, los objetivos y las palancas de acción de cada mente.

- Definir un plan de acción para comenzar la carrera profesional

Los programas de mentoring se han venido desarrollando en la ETSIME desde el año 2010 a través de varios proyectos:

- Proyecto Mentor: alumnos de últimos cursos mentorizan y guían a los alumnos de reciente incorporación a la ETSIME.

- Proyecto Enseña: alumnos de la ETSIME mentorizan y guían a estudiantes de secundaria.

Por tanto, había una necesidad de realizar un mentoring profesional a los estudiantes para ayudarles a su incorporación en el mercado de trabajo y así completar las actividades de mentoring de la ETSIME: de estudiante a recién llegado, de estudiante a posible futuro estudiante, de profesional a estudiante.

\section{DESCRIPCIÓN}

La actividad se realizó desde noviembre de 2020 hasta abril de 2021. Inicialmente se lanzó una campaña de publicidad de la actividad a través de redes sociales (linkedin, twitter, Instagram y web de la ETSIME) y se programó una primera sesión informativa en formato de webinar donde las coordinadoras de la actividad informaron sobre la misma y resolvieron las preguntas de los estudiantes interesados.

El programa comenzó con un total de 8 estudiantes, 4 mujeres y 4 hombres, de los cuales 7 eran estudiantes de grado y 1 de máster. Las mentoras, una por cada estudiante, es decir, un total de 8 , eran todas profesionales del sector energético y miembros de AEMENER. La gestión de todo el proceso se llevó a cabo desde la ETSIME mediante un equipo de TEAMS con varios canales para poder informar de forma conjunta o separada a mentoras y mentees.

El proceso de emparejamiento de mentoras y mentees se llevó a cabo enviando un cuestionario inicial para conocer el perfil de los mentees. El cuestionario se realizó por medio de la herramienta Microsoft Forms y constaba de 20 preguntas relativas a:

- Título que se está cursando, formación (ya sea de ingeniería o de otro tipo), hobbies y campos de interés personales.

- Participación previa en procesos de mentoring o similares

- Motivaciones, objetivos, prioridades, áreas a mejorar y expectativas respecto al programa

- Valores que debe tener la relación con tu mentor.

Una vez realizado el emparejamiento, dieron comienzo las sesiones individuales con la mentora. El mentee inicia este proceso estableciendo en un documento escrito su visión y objetivos. Estas sesiones individuales serán establecidas en el horario y fecha que cada pareja mentor-mentee establezca. Lo tratado en estas sesiones, por razones de confidencialidad no será abordado en este artículo.

Con objetivo de complementar la formación y la información de los mentees, se realizaron las tres sesiones grupales siguientes:

- Sesión on-line informativa sobre Másteres: impartida por el subdirector de posgrado y el director de la ETSIME. En ellas se abordaron aspectos generales sobre el marco legal de los másteres, la diferencia entre un máster habilitante con respecto a uno que no lo es y se expuso la oferta de posgrado de la ETSIME. Además, se atendieron preguntas tanto por parte de los mentees como por parte de las mentoras que desconocían los detalles relativos a los planes de Bolonia. Fue una sesión donde hubo mucha participación y tuvo una duración de unas dos horas y media.

- Sesión on-line sobre Comunicación Eficaz: impartida por una de las mentoras conjuntamente con su mentee. En la sesión se abordaron aspectos generales sobre comunicación y en último lugar se reestudiaron dos casos de comunicación por medio de un role play entre la mentora y el mentee. Los mentees participaron comentando las dos situaciones expuestas y señalando aquellos errores de comunicación que observaron.

- Sesión on-line “Conoce tu vocación y mejora tu empleabilidad": impartida por Catalina Díaz Freire, profesional del sector de los recursos humanos y con amplia experiencia en la selección de perfiles de ingeniería. En esta sesión se comentaron las competencias que son más demandadas en el actual mercado laboral y se señalaron varios tests que los estudiantes podrían hacer on-line para conocer mejor sus habilidades.

Las sesiones individuales mentor-mentee fueron concluyendo a lo largo del mes de abril. El 7 mayo de 2021 se realizó la entrega de diplomas presencial a la que 
asistieron las mentoras y sus mentees y con ello se concluyó la actividad durante el curso 2020/21.

\section{RESUltados}

Con el objetivo de evaluar la actividad y la satisfacción tanto de mentoras como de mentees, se elaboraron dos encuestas anónimas, una para los mentees y otra para las mentoras.

Las encuestas se elaboraron con el software Microsft Forms y parte de los resultados se muestran en las gráficas que se encuentran a lo largo de esta sección.

En cuanto a la satisfacción de las mentoras, los resultados se presentan en la Figura 1, Figura 2 y Figura 3.

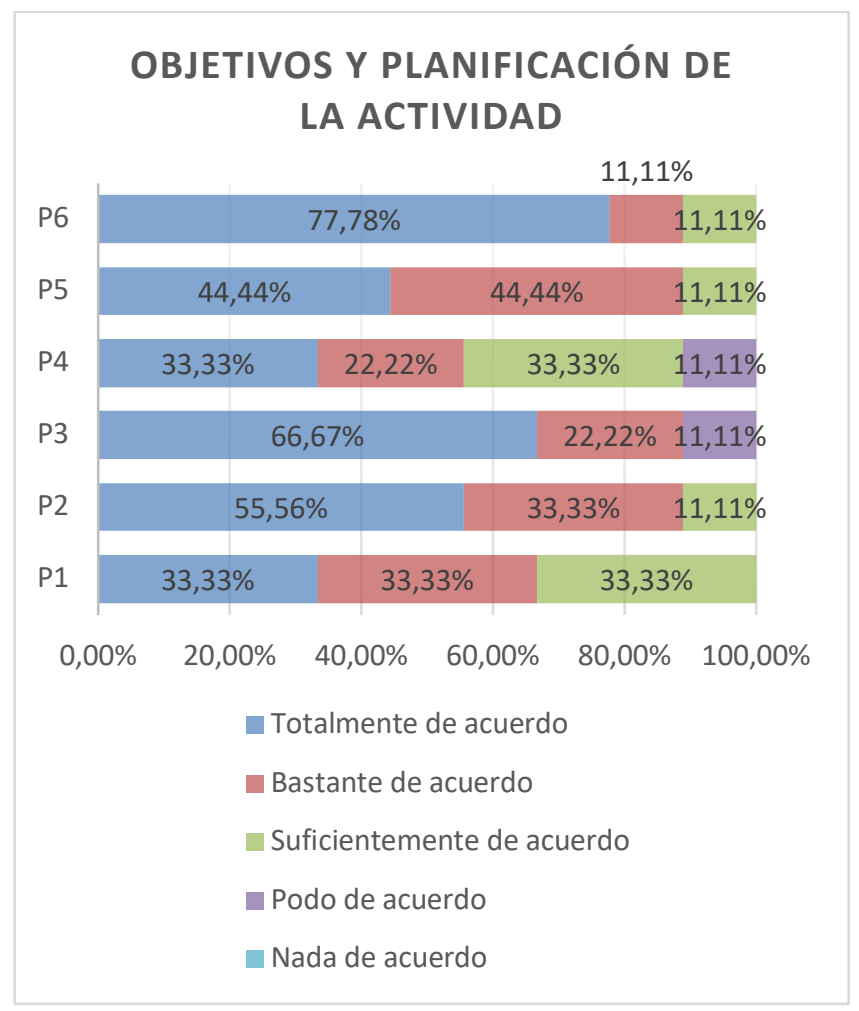

Figura 1: Resultados de la encuesta a las menotres respecto a los objetivos y planificación de la misma.

Donde las preguntas son:

P1: Está bien diseñada y organizada

P2: Se ha podido cumplir la planificación prevista

P3: La duración ha sido adecuada en relación a las necesidades de mi mentee

P4: He dispuesto de los suficientes medios para el desarrollo de la actividad mentora

P5: Se han cumplido mis objetivos con respecto al programa

P6: El coordinador me ha prestado ayuda siempre que lo he necesitado.

Como se puede apreciar la mayor parte de mentoras están de acuerdo o bastante de acuerdo en las preguntas planteadas, salvo en la pregunta $\mathrm{P} 4$, donde se observa que un $42 \%$ de las mentoras considera que está sufientemente de acuerdo o poco de acuerdo en cuanto a si ha tenido los medios necesarios para realizar su labor.

\section{EN CUANTO A LA RELACIÓN CON MI MENTEE}

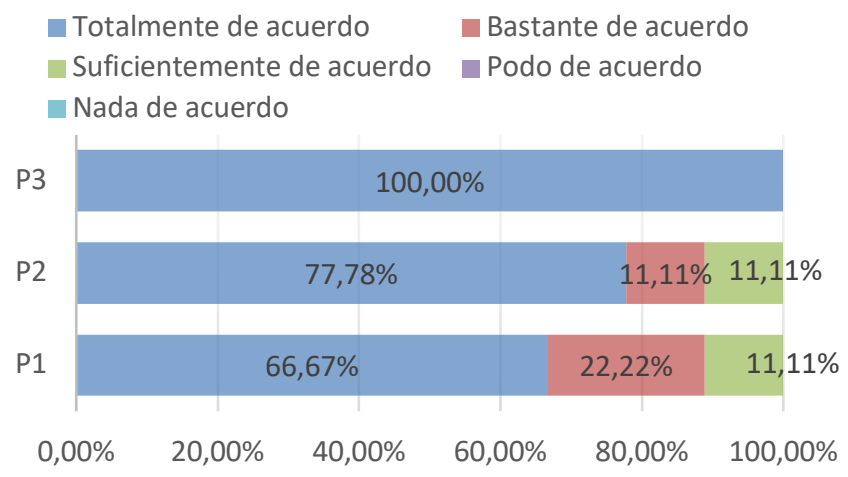

Figura 2: Resultados de la encuesta a las mentoras respecto a la relación con los mentees.

Donde las preguntas son:

P1: La relación con el mentee ha sido satisfactoria

P2: El mentee ha colaborado en las acciones propuestas

P3: Se ha mantenido la confidencialidad.

A la vista de los resultados, la elación con los mentees ha sido muy satisfactoria.

Por último, respecto a la valoración general de la actividad, las respuestas se muestran en la Figura 3, donde se pone de manifiesto un alto de grado de satisfacción.

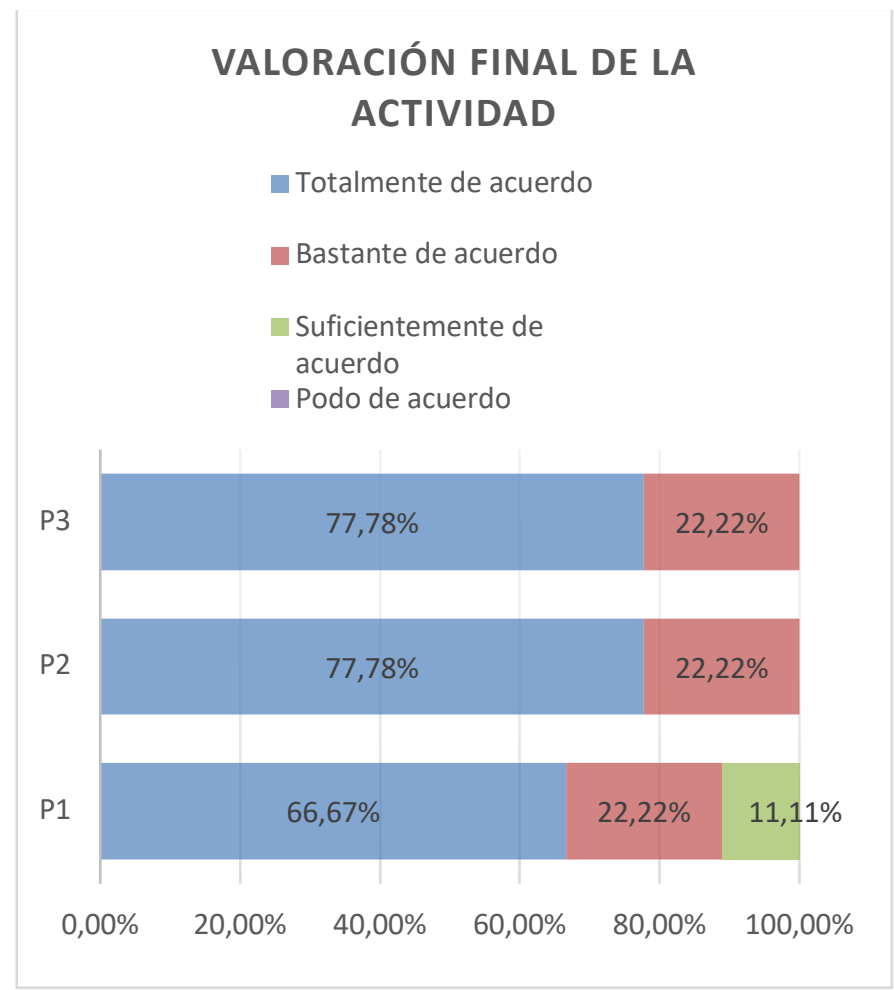

Figura 3: Resultados de la encuesta a las mentoras respecto a la valoración final de la actividad

Siendo: 
P1: Recomendarías el programa a un amigo para que actuara como mentor

P2: Volvería a participar en un programa de este tipo

P3: En general, estoy satisfecho/a con el desarrollo de la actividad

En cuanto al tiempo dedicado, el $50 \%$ ha dedicado unas 10 horas, el $37 \% 20$ horas y un $13 \%$ ha dedicado 30 horas aproximadamente.

Sobre si es necesario añadir alguna sesión sobre alguna temática específica, las respuestas abiertas han sido:

- Cómo buscar trabajo en el entorno actual/ orientación profesional/tendencias de mercado/internalización

- Habilidades de comunicación en el entorno laboral

Hay bastantes observaciones relativas a la homogenización de la metodología para que todas las mentoras utilicen una sistemática parecida.

En cuanto a la satisfacción de los mentees, los resultados se muestran en la Figura 4, Figura 5, Figura 6 y Figura 7.

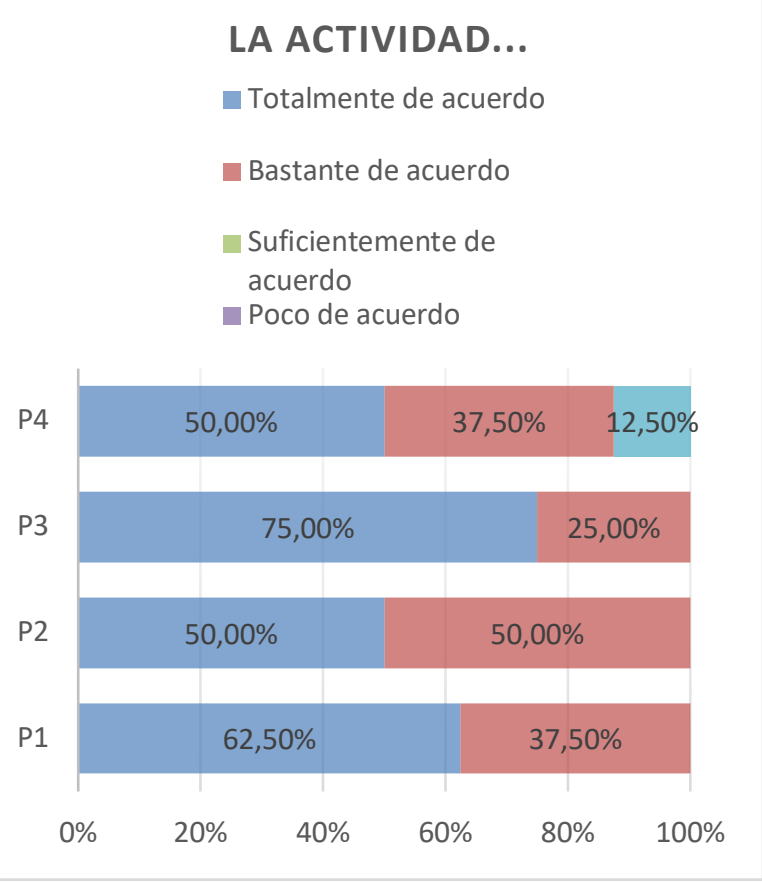

Figura 4: Resultados de la encuesta a los mentees respecto a la planificación de la actividad

P1: está bien diseñada y organizada

P2: te ha orientado en aspectos académicos

P3: te ha orientado en aspectos laborales

P4: te ha ayudado a reforzar tu autoestima

\section{RESPECTO A LOS OBJETIVOS DE LA ACTIVIDAD...}

- Totalmente de acuerdo

Bastante de acuerdo

- Suficientemente de acuerdo

- Poco de acuerdo

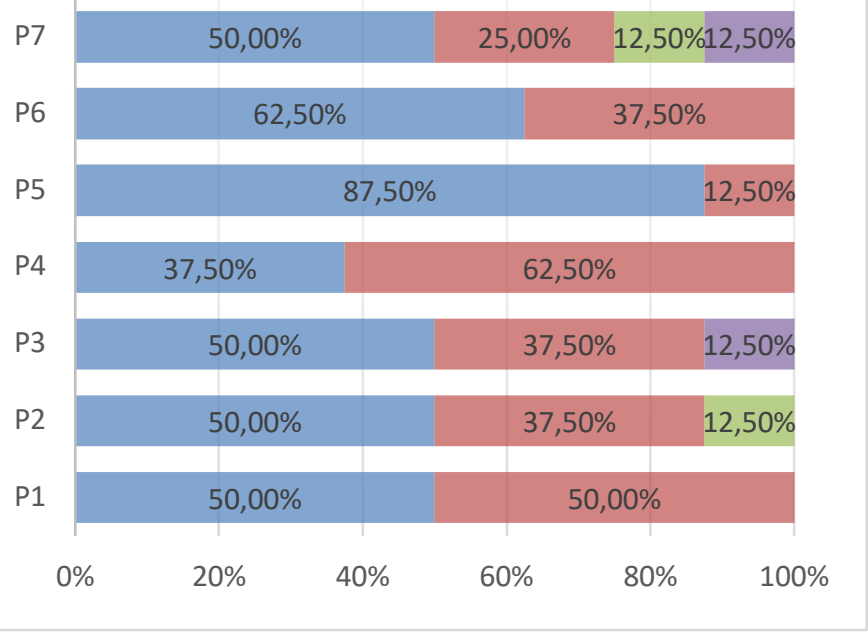

Figura 5: Resultados de la encuesta a los mentees respecto a los objetivos de la actividad.

P1: Se ha cumplido la planificación prevista

P2: El planteamiento o enfoque ha sido el correcto

P3: Se han cumplido tus objetivos con respecto al programa

P4: Has podido identificar un objetivo ambicioso pero alcanzable.

P5: Se han lanzado nuevas ideas

P6: Se ha contribuido a realizar un cambio de perspectiva

P7: La duración del programa ha sido adecuada en relación con mis necesidades 


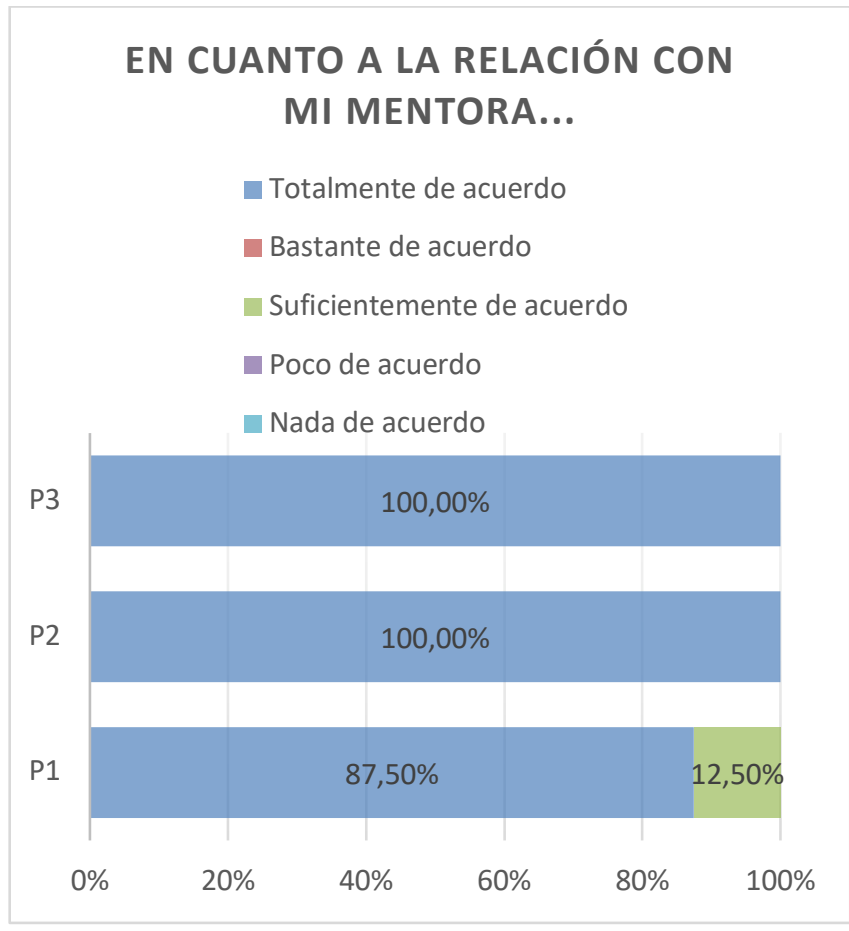

Figura 6: Resultados de la encuesta a los mentees respecto a la relación con su mentora.

P1: El apoyo recibido por mi mentora ha sido satisfactorio

P2: Se ha creado un vínculo honesto y sincero con mi mentora

P3: Se ha mantenido la confidencialidad

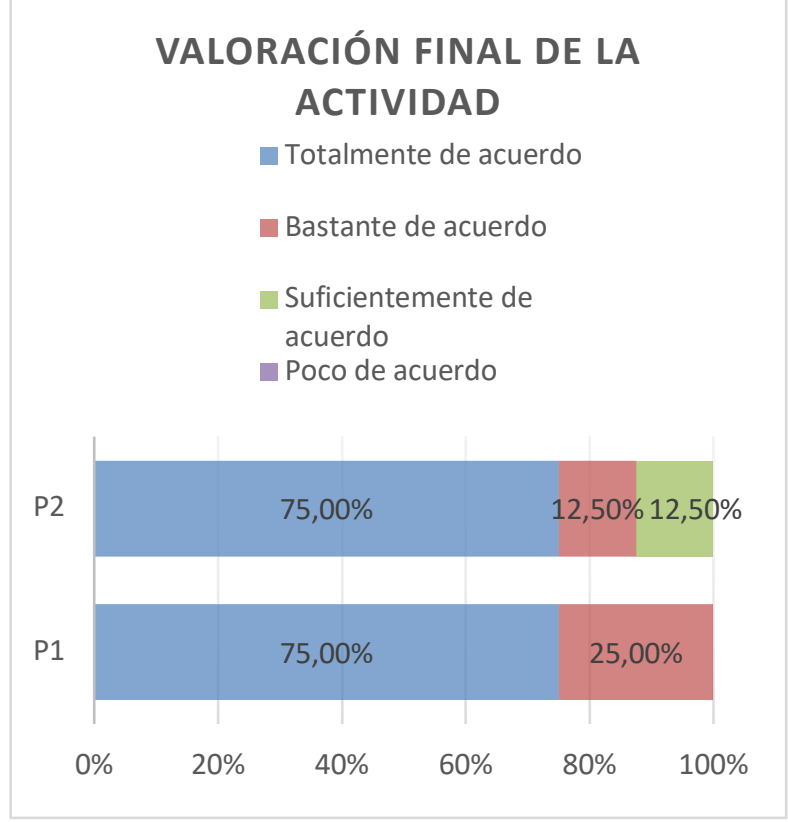

Figura 7: Resultados de la encuesta a los mentees respecto a la valoración de la actividad.

P1: Recomendarías el programa a un amigo/compañero de clase

P2: En general, estoy satisfecho/a con el desarrollo de la actividad.
En cuanto a las horas dedicadas, hay un $50 \%$ de los estudiantes que han dedicado entre 20 y 30 horas, un $25 \%$ que han dedicado entre 40 y 50 , un $12,5 \%$ que ha dedicado 10 horas y otro $12,5 \%$ que no sabe cuántas horas ha dedicado.

En general, los mentees muestran un alto grado de satisfacción, aunque hay un $25 \%$ que está suficientemente de acuerdo y poco de acuerdo en que la duración del programa ha sido adecuada.

Los mentees comentan en las preguntas abiertas que sería bueno añadir en la actividad alguna sesión específica sobre soft skills, sobre cómo afrontar su primera entrevista de trabajo e incluso sobre autoestima, motivación, comunicación y superar la frustración.

Los mentees han aportado su opinión de forma abierta de la siguiente manera:

"Esta actividad me ha aportado mucho valor. Espero que las mentoras también estén contentas con el programa y pueda continuar en futuras ediciones".

"Me gustó mucho la sesión de comunicación eficaz y la de los distintos tipos de másteres me resultó muy interesante. Estoy muy contenta por haber participado en esta actividad, me ha servido mucho".

"Muy buena iniciativa y muy agradecido por todo lo que he aprendido de los mentores".

"Me parece que es un curso bastante completo y que ayuda un montón, a mí incluso se me ha hecho corto!. Lo que estaría bien es hacer más sesiones del grupo entero e intercambiar sensaciones y opiniones. ¡Gracias por ofrecernos esta gran oportunidad!"

"Me gustó mucho esta experiencia, siento que esta son el tipo de actividades complementarias que todo alumno debería vivir. Me abrió los ojos en muchos aspectos, siento más seguridad y ahora tengo más herramientas para enfrentar la vida tanto personal como profesionalmente. Gracias por brindarnos esta oportunidad."

"Me ha parecido una idea muy buena, personalmente me ha ayudado un montón, hasta mi madre está contenta con que haya participado en el proyecto. Las sesiones en grupo han sido muy útiles y super amenas. Simplemente agradecer que se haya organizado este proyecto de mentoring, coordinarlo tiene un trabajo detrás que no se ve y que requiere dedicación, así que muchas gracias!!"

"Creo que podría ser beneficioso para los alumnos que las mentoras pudieran optar a una orientación previa sobre cómo reforzar la autoestima, motivación, comunicación, apoyo o sobre cómo ayudar a mejorar la percepción de las personas sobre sí mismas. Quizá involucrando a una persona que haya estudiado psicología, no lo sé. Son unas mentoras maravillosas pero en ese ámbito yo he sentido que me hubiera gustado algo más. También me gustaría dar las gracias a la coordinadora de la actividad por ofrecernos esta oportunidad; por su organización, su trabajo y su entusiasmo. 


\section{CONCLUSIONES}

La actividad "Mentoring con energía" se ha realizado con éxito en la ETSIME durante el curso 2020/21. La continuidad del programa está garantizada y muy probablemente se aumentará el número de parejas mentee/mentor para próximas ediciones. Se recomienda para futuras ediciones mayor número de sesiones grupales y a poder ser en formato presencial. Los mentees señalan que sería conveniente reforzar las soft skills con alguna sesión específica. Además, de cara a homogeneizar la sistemática de las mentoras, es recomendable potenciar la comunidad de mentoring de AEMENER, donde se vayan añadiendo materiales, experiencias de otros años, manuales de buenas prácticas, etc.

La actividad ha sido muy enriquecedora en general para las mentoras, los mentees y para la ETSIME, ya que con ella se refuerzan los lazos con la empresa privada y se mantiene un vínculo directo con el sector energético.

\section{AGRADECIMIENTOS}

Los autores de este trabajo destacan el agradecimiento mutuo de las dos instituciones a AEMENER y a ETSIME, que han hecho posible que se pudiera llevar a cabo y, en particular, a las mentoras la participación y el entusiasmo con que han abordado esta actividad.

\section{REFERENCIAS}

DiRenzo, M. S., Linnehan, F., Shao, P., \& Rosenberg, W. L. (2010). A moderated mediation model of e-mentoring. Journal of Vocational Behavior, 76(2), 292-305. doi:https://doi.org/10.1016/j.jvb.2009.10.003

Risquez, A., \& Sanchez-Garcia, M. (2012). The jury is still out: Psychoemotional support in peer e-mentoring for transition to university. The Internet and Higher Education, 15(3), 213-221. doi:https://doi.org/10.1016/j.iheduc.2011.11.003

Tinoco-Giraldo, H., Torrecilla Sánchez, E. M., \& GarcíaPeñalvo, F. J. (2020). E-mentoring in higher education: A structured literature review and implications for future research. Sustainability, 12(11), 4344. 